\title{
Point-of-Care Testing Using Three Dimensional Optical Biosensor Based on Microfluidic Technology
}

\author{
Chunxiu Liu*, Haoyuan Cai, Jian Jia, Tianyang Cao, Tong Li, Tianjun Ma, Chang Liu* \\ State Key Laboratory of Transducer Technology, Institute of Electronics, Chinese Academy of Sciences, Beijing, China \\ Email: ^cxliu@mail.ie.ac.cn, tuengineer@qq.com
}

How to cite this paper: Liu, C.X., Cai, H.Y., Jia, J., Cao, T.Y., Li, T., Ma, T.J. and Liu, C. (2016) Point-of-Care Testing Using Three Dimensional Optical Biosensor Based on Microfluidic Technology. Journal of Biosciences and Medicines, 4, 56-61. http://dx.doi.org/10.4236/jbm.2016.412009

Received: November 2, 2016 Accepted: November 24, 2016 Published: December 1, 2016

\begin{abstract}
We have presented a three dimensional optical protein chip that fulfills the demanding for point-of-care diagnostics in terms of ease-of-use (one step assay), miniaturization $(5 \mu \mathrm{l})$. The artful combination of magnetic nanoparticles on chip and total internal reflection imaging (TIRI) technology permits the sensitive and rapid detection of hs-CRP (high-sensitivity C-reactive protein). The whole test was complete within $10 \mathrm{~min}$ using "all in one step" assay with a limit of detection of $0.1 \mathrm{ng} / \mathrm{mL} \mathrm{hs}-\mathrm{CRP}$. The measuring range for hs-CRP could be extended to $10 \mathrm{ng} / \mathrm{mL}$. The chip can also be used to detect more parameters in blood samples.
\end{abstract}

\section{Keywords}

Point-of-Care Testing (POCT), Three Dimensional Optical Chip, hs-CRP

\section{Introduction}

This POCT (point-of-care testing) has the advantages of simple equipment operation, accurate and reliable results, those novel technologies and devices to all visitors with a new concept, namely "A number of measurement results can be obtained in place close to the patients in a very short period of time". POCT is a new field of medical development, achieving the rapid progress with the development of high technology and comprehensive application, conforming to the current, fast-paced work of the people [1][5]. The patient can obtain timely diagnosis and treatment.

The development of microfluidic technology provides a new idea and platform for the rapid and high-throughput detection of protein, and greatly reduces the cost of detection. Compared with the traditional analysis method, the microfluidic detection technology has the advantages of high efficiency, fast, less reagent consumption, auto- 
mation and so on.

Study on POCT microfluidic chip challenged the detection of minimal volume, complex pretreatment samples. The precision and efficiency of the real-time diagnostic test and its device is an important change in the field of modern life and health. The microfluidic detection and analysis chip is the main technology to make real-time diagnosis accurate and effective [6] [7] [8] [9].

Total internal reflection imaging (TIRI) technology as a branch of optical detection combined with the microfluidic chip can be used for the detection and analysis of a variety of substances [10] [11] [12]. The detection is based on COMS camera capture of the reflected light of the chip reaction interface. This work developed the fast POCT detection of low concentration hs-CRP protein by three dimensional microfluidic chip and total internal reflection imaging (TIRI).

\section{Materials and Methods}

\subsection{Experimental Materials}

A pair of commercially available sandwich anti-CRP monoclonal antibodies ( $\mathrm{mAb}$ ) was purchased from Abzymo Biosciences Co. (Beijing, China). The tracer monoclonal antibody was functionalized with biotin. The Carboxyl Streptavidin Magnetic Particles (500 nm diameter, $0.5 \% \mathrm{w} / \mathrm{v}$ ) were purchased from ACME microspheres Co. (Indianapolis, IN, USA). Polylysine (PLL, P4832, Sigma), BSA and other reagents were purchased from Sigma-Aldrich Inc. (St. Louis, MO, USA). The buffer solutions were prepared with ultra-pure water. Concentration values stated in this study are used by serial dilutions in PBS buffer.

\subsection{Experimental Equipment}

The Poly-Pico System (PolyPico., Ireland) device was used to precisely and uniformly dispense $\mathrm{pL}$-sized arrayed capture monoclonal antibodies particles. The high precision laser engraving machine DPSS (Suzhou, China) can be used to cut double sided adhesive tape to form the microfluidic channel, and reaction chamber of the chip. The TIRI home made system containing the optics module and the magnetic module was used to take image and collect data.

\subsection{Fabrication of the 3D Optical Biosensor}

The optical chip was composed of a 3D glass bottom board, double-sided adhesive on the micro channel, and cover sheet (Figure 1). The basic structure of the micro channel was cut by the laser cutting machine on the double-sided adhesive, and the double-sided adhesive was pasted on the surface of the $3 \mathrm{D}$ glass base plate. The trace $\mathrm{mAb}$ and capture $\mathrm{mAb}$ were immobilized on the top and bottom of the reaction chamber. The chip can be divided into the sample port, reaction and detection zone, time gate and waste reservoir.

\subsection{Detection Method and Data Analysis Method}

The principle of detection of the 3D TIRI optical chip is a one-step sandwich immu- 


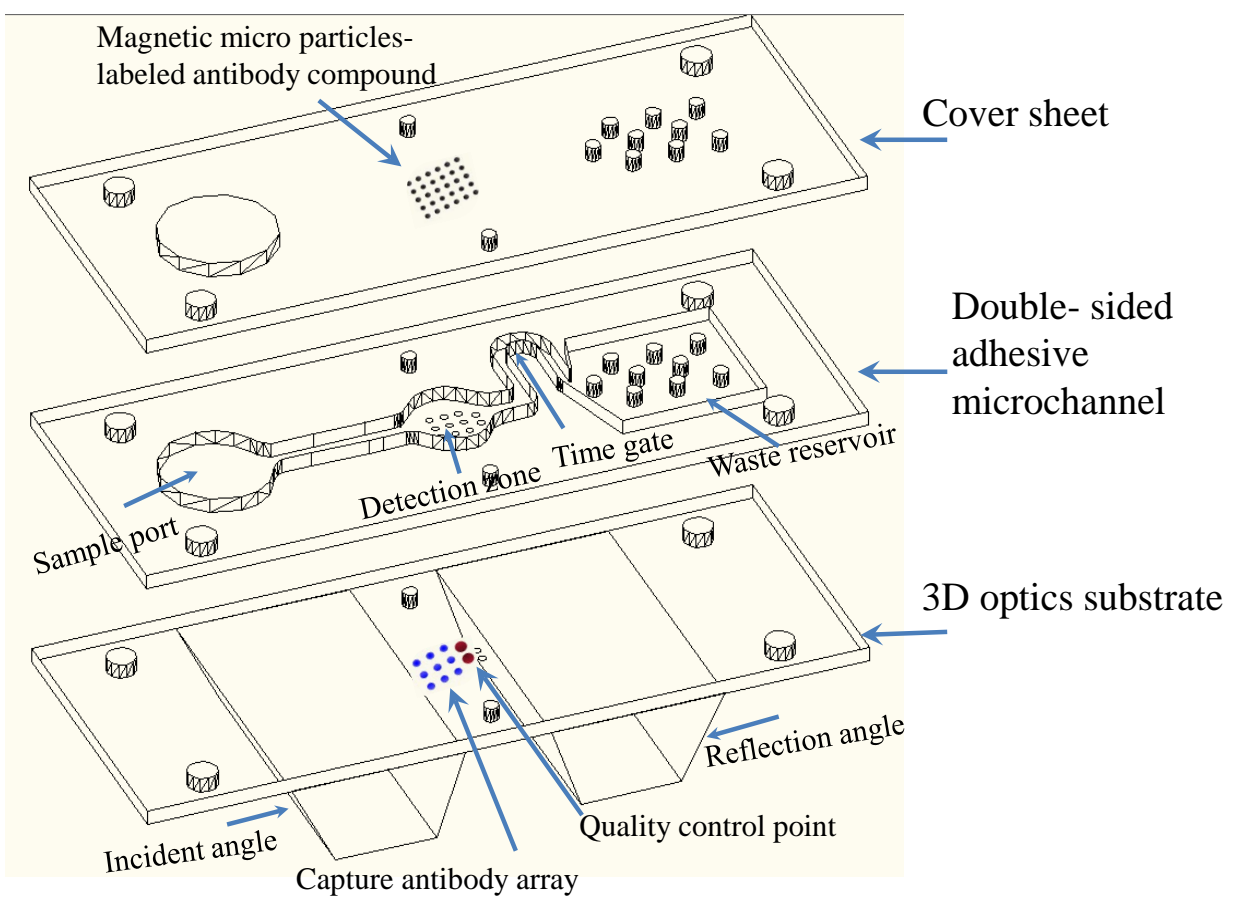

Figure 1. The schematic illustration and assembly of 3D optical TIRI chip.

noassay method shown in Figure 2. When the samples were added, the sample rehydrated the trace $\mathrm{mAb}$ and magnetic particle in the detection area and then the antigen action with the composites. The bidirectional magnetic force was open and the capture antibody immobilization in the bottom acted with the Mag-mAb-Ag complex and form magnetic spots at the bottom of the reaction area. The total internal optical signals are subject to magnetic speckle interference, and the signal was inverse correlation with the antigen concentrations. Matlab software was used to assay the obtained magnetic spot imaging.

\section{Results}

\subsection{Optimization on the Reflection Angle of the 3D Chip}

One major aim of the assay development was the capture of the clear images that occurred during one-step sandwich immunoassay. The incidence angle and reflection angle of the 3D optical chip was symmetric in the center of the reaction zone. The incidence angle should meet the conditions of total internal reflection imaging according to the refractive index of the material of the 3D chip. The angular range of the reflection angle has important influence on imaging quality. When the reflection angle continues to increase, the image sharpness decreased rapidly with the increasing angle of reflection. The image changed from circles to ellipse when the angle gradually increased and the image distortion aggravated when the angle reached 70 degree angle (Figure 3). It was too small to form a total internal reflection if the reflection angle is less than 45 degree. 


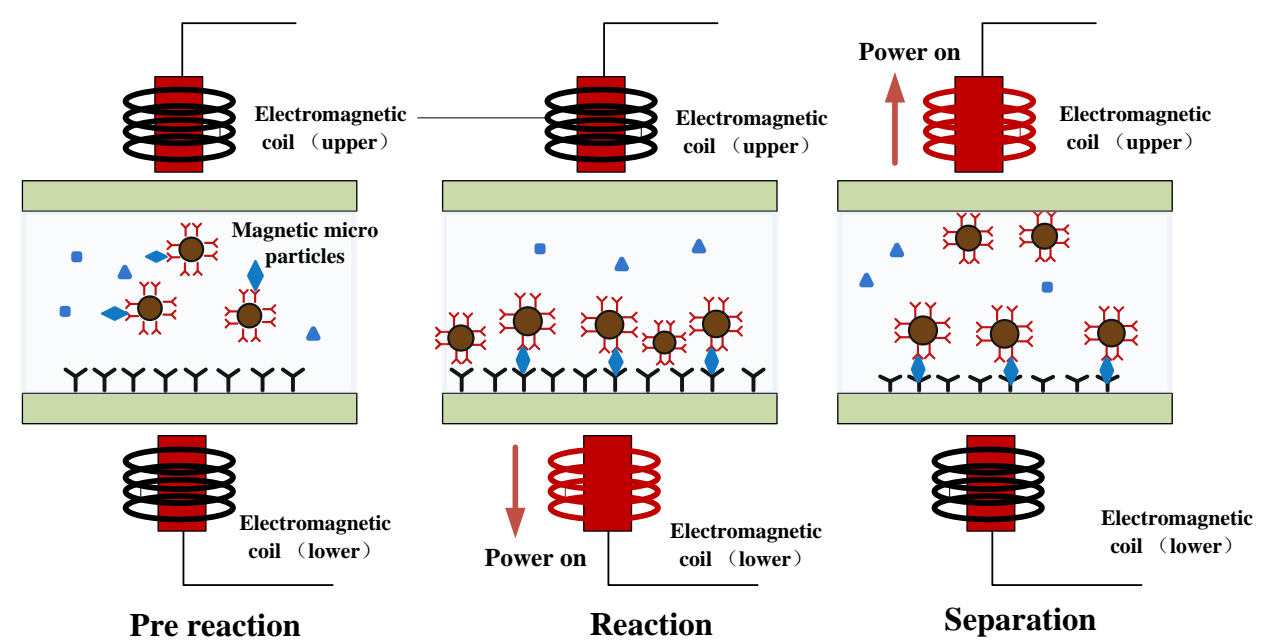

Figure 2. Schematic diagram of detection principle of sandwich immunoassay method.

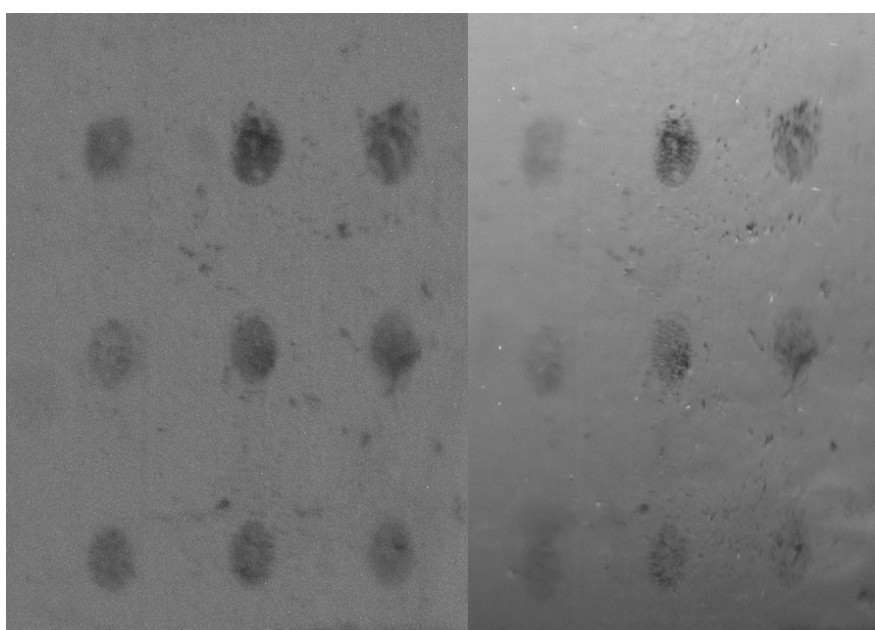

Angle of 45
Angle of 60

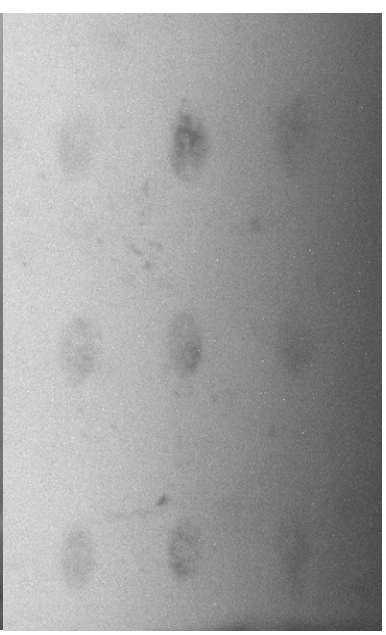

Angle of 70

Figure 3. The TIRI image of $3 \mathrm{D}$ chip at different reflection angle.

\subsection{Detection of hs-CRP}

The standard curves with a dilution series of standard CRP samples by PBS buffer were created. The assay achieved an LOD value of $0.1 \mathrm{ng} / \mathrm{mL}$ in standard CRP samples. The linear fits to the curves are shown in Figure 4. The sensitivity of the assay is sufficient to distinguish hs-CRP levels during $0.1-10 \mathrm{ng} / \mathrm{mL}$ (Figure 4). To enable the measurement of lower and higher concentrations of CRP, further detection and improvements to the chip system are necessary. The assay required no sample preparation and is therefore easy to handle, which is suitable for POC measurement at the patient.

\section{Conclusion}

A new, highly sensitive assay to quantify CRP via a POCT device was developed. The device fulfills all the important characteristics of a POCT application. Our assay has a very short measurement time of less than $10 \mathrm{~min}$, and it requires only a small sample 


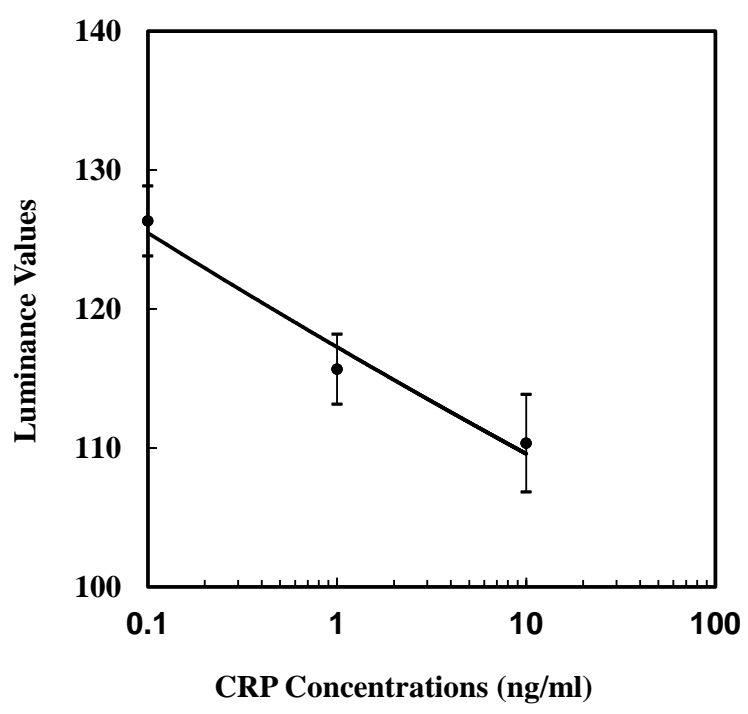

Figure 4. Standard curves of CRP with the sandwich assay using paired monoclonal antibody, number of concentrations: 3 , number of repeats: 3 .

volume $(20 \mu \mathrm{L})$. There is no need for sample pretreatment because the device uses ready to-use 3D optical chip. Furthermore, it is simple and safe to handle. Furthermore, the assay operates in the necessary sensitivity range $(0.1-10 \mathrm{ng} / \mathrm{mL})$ with standard samples.

\section{Acknowledgements}

This work is supported by the NSFC (No.60801032, 61271147, 61372052), the major national scientific research plan (2011CB933202), the project of CAS (KFJ-EW-STS$046)$ and the high resolution major projects (06-Y30B04-9002-13/15).

\section{References}

[1] Natalia, L.B., Jorge, D.G. and Johann, F.O. (2016) The Future Point-of-Care Detection of Disease and Its Data Capture and Handling. Anal Bioanal Chem, 408, 2827-2837. http://dx.doi.org/10.1007/s00216-015-9249-2

[2] Jung, W., Han, J., Choi, J.W. and Ahn, C.H. (2015) Point-of-Care Testing (POCT) Diagnostic Systems Using Microfluidic Lab-on-a-Chip Technologies. Microelectronic Engineering, 132, 46-57. http://dx.doi.org/10.1016/j.mee.2014.09.024

[3] Ouellette, A.L., Li, J.J., Cooper, D.E., Ricco, A. and Kovacs, G.T.A. (2009) Evolving Pointof-Care Diagnostics Using Up-Converting Phosphor Bioanalytical Systems. Analytical Chemistry, 81, 3216-3221. http://dx.doi.org/10.1021/ac900475u

[4] Luppa, P.B., Müller, C., Schlichtiger, A. and Schlebusch, H. (2011) Point-of-Care Testing (POCT): Current Techniques and Future Perspectives. Trac Trends in Analytical Chemistry, 30, 887-898. http://dx.doi.org/10.1016/j.trac.2011.01.019

[5] Yang, Z. and Zhou, D.M. (2006) Cardiac Markers and Their Point-of-Care Testing for Diagnosis of Acute Myocardial Infarction. Clinical Biochemistry, 39, 771-780. http://dx.doi.org/10.1016/j.clinbiochem.2006.05.011 
[6] Pfäfflin, A. and Schleicher, E. (2009) Inflammation Markers in Point-of-Care Testing (POCT). Analytical \& Bioanalytical Chemistry, 393, 1473-1480. http://dx.doi.org/10.1007/s00216-008-2561-3

[7] Kiechle, L.F. (2015) Three POCT Molecular Applications: A View into Future Development, Point of Care. The Journal of Near-Patient Testing \& Technology, 14, 95-98. http://dx.doi.org/10.1097/poc.0000000000000052

[8] Baldini, F., Bolzoni, L., Giannetti, A., et al. (2009) A New Procalcitonin Optical Immunosensor for POCT Applications. Analytical and Bioanalytical Chemistry, 393, 1183-1190. http://dx.doi.org/10.1007/s00216-008-2547-1

[9] Rascher, D., Geerlof, A., Kremmer, E., Krämer, P. and Schmid, M. (2014) Total Internal Reflection (TIRF)-Based Quantification of Procalcitonin for Sepsis Diagnosis-A Pointof-Care Testing Application. Biosensors \& Bioelectronics, 59C, 251-258.

http://dx.doi.org/10.1016/j.bios.2014.03.052

[10] Mejri, M.B., Baccar, H., Ktari, T., Aouni, M. and Abdelghani, A. (2011) Detection of E. coli Bacteria Using Impedance Spectroscopy and Surface Plasmon Resonance Imaging Based Biosensor. Sensor Letters, 9, 1-3. http://dx.doi.org/10.1166/sl.2011.1747

[11] Michael, J.O., Victor, H.P., Brueck, S.R.J. and Gabriel, P.L. (2001) A Surface Plasmon Resonance Array Biosensor Based on Spectroscopic Imaging. Biosensors \& Bioelectronics, 16, 97-108. http://dx.doi.org/10.1016/S0956-5663(00)00137-8

[12] Tan, H.M., Pechprasarn, S., Zhang, J., Pitter, M.C. and Somekh, M.G. (2016) High Resolution Quantitative Angle-Scanning Widefield Surface Plasmon Microscopy. Scientific Reports, 6, Article Number: 20195. http://dx.doi.org/10.1038/srep20195

\section{Submit or recommend next manuscript to SCIRP and we will provide best service for you:}

Accepting pre-submission inquiries through Email, Facebook, LinkedIn, Twitter, etc. A wide selection of journals (inclusive of 9 subjects, more than 200 journals)

Providing 24-hour high-quality service

User-friendly online submission system

Fair and swift peer-review system

Efficient typesetting and proofreading procedure

Display of the result of downloads and visits, as well as the number of cited articles

Maximum dissemination of your research work

Submit your manuscript at: http://papersubmission.scirp.org/

Or contact jbm@scirp.org 